Aus der chirurgischen Iniversitätsklinik in Rostock.

\title{
Kritisch-statistische Bemerkungen zur Frage der Gastroenterostomia anterior antecolica.
}

Von Dr. med. Egbert Schwarz, I. Assistenten der Klinik.

Die Frage über die Brauchbarkeit der Gastroenterostomie bzw. der einen oder anderen Methode derselben scheint in den letzten Io - 20 Jahren dahin entschieden worden zu sein, daß die vordere Gastroenterostomie nur in seltenen Fällen ausgeführt werden soll, während im allgemeinen da, wo eine Gastroenterostomic überhaupt angezeigt erscheint, der Vorzug der v. Hackerschen Methode zu geben ist. Diese jetzt wohl von den meisten Chirurgen geteilte Ansicht hat auch in den neueren Lehr- und Handbüchern, so durch Bie ${ }^{1}$ ), Kausch ${ }^{2}$ ) und Schloffer ${ }^{3}$ ) Ausdruck gefunden. Die Vorteile und Nachteile der verschiedenen Methoden der Gastroenterostomie werden an den entsprechenden Stellen hervorgehoben, und überall kommt die Ansicht zum Ausdruck, daß die v. Hackersche hintere Gastroenterostomie im allgemeinen vorzuziehen sei.

Die Verhandlungen der Deutschen Gesellschaft für Chirurgie im Jahre I9I 2 haben ergeben, daß die Vorteile und Nachteile der einen oder anderen Methode der Gastroenterostomie doch nicht von allen. Chirurgen in gleicher Weise gewertet werden, und daß es doch noch Chirurgen gibt, die die alte Wölflersche Methode bevorzugen. In diesem Sinnc teilte W. M üller $\mathrm{r}^{4}$ ) im Anschluß

I) Bier. Chirurgische Operationslehre von Bier, Braun und Kümmell, Bd. 3, S. 102-II 4 .

2) Kausch, Chirurgic des Bauches, im Handbuch der prakt. Chir. von v. Bruns, Garrè und Küttner, Bd. 3, S. 143-1 $4^{8}$.

3) Schloffer. Lehrbuch d. Chir., herausgeg. von Wullstein u. Wilms, Bd. 2, S. 8 o.

4) Mi iller. W. Verhandl. d. Dentschen Ges. f. Chir. I9I2, 4I. Kongree, Bil. I, S. r7o/So. 
an einen von $A$. Kocher ${ }^{1}$ ) auf dem erwähnten KongreB gehaltenen Vortrag über „Ulcus ventriculi und Gastroenterostomie“ "seine guten Erfahrungen mit der vorderen Gastroenterostomie auf Grund von annähernd 200 Operationen mit, in deren Verlauf eine ernstliche Komplikation, insbesondere ein typischer Circulus vitiosus nur sehr selten beobachtet werden konnte. Er sei deshalb auch jetzt noch Anhänger der vorderen Gastroenterostomie geblieben. Dieser Ansicht schlossen sich auch noch andere Fachgenossen an, so unter andern Borchard ${ }^{2}$ ) und $\mathrm{Schlange}{ }^{3}$ ) Auch Enderlen äußerte sich dann privatim zugunsten der Gastr. ant. Sie hätten nachteilige Erfahrungen mit der vorderen Gastroenterostomie nicht gemacht und seien deshalb mit gewissen Einschränkungen, die auch $M \ddot{u} 11$ e r zugibt, Anhänger der Wölf ler schen Methode geblieben.

Die Tatsache dieser noch immer geteilten Meinungen in der Gastroenterostomiefrage gab mir Veranlassung, die Erfahrungen der hiesigen Klinik mit der in Frage stehenden Operationsmethode so weit möglich zu sammeln. Während in den Arbeiten der neueren Zeit über Magenchirurgie oft mehr die Frage „Resektion oder Gastroenterostomie“, besonders bei der Behandlung des Magengeschwürs, diskutiert wurde, soll im vorstehenden des näheren nur darauf eingegangen werden, ob tatsächlich, wie von vielen Seiten behauptet wird, die vordere Gastroenterostomie wegen der in ihrem Anschluß möglichen Komplikationen als eine zu gefährliche Methode anzusehen ist und wie die Gefahren der Gastroenterostomie selbst zu beurteilen sind.

Bekanntlich lassen sich die Komplikationen im wesentlichen in drei Punkten zusammenfassen. Es besteht

I. die Gefahr eines Ulcus pepticum jejuni, welches bei der vorderen Gastroenterostomie häufig vorkommen soll und durch die hintere Gastroenterostomie eher vermieden wird;

2. in der Gefahr eines Circulus vitiosus, welcher durch Spornbildung, Kompression der abführenden Jejunumschlinge zwischen

I) Kocher, A., Über Ulcus ventriculi und Gastroenterostomie. Verhandl. d. Deutschen Ges. f. Chir. 19I2, 4I. KongreB, Bd. 2, S. 415.

2) Borchard. Verhandl. d. Deutschen Ges. f. Chir. I9r2, 4I. Kon. greß, Bd. I, S. I79/80.

3) Schlange, ebenda. 
Magen und zuführender Schlinge, anisoperistaltische Lagerung der Gastroenterostomieschlinge und Atonie des Magens entsteht;

3. in der Gefahr einer Kompression des Colon transversum durch eine zu kurz gewählte Jejunumschlinge oder Kompression der Dündarmschlinge durch das geblähte Colon transversum oder ein sehr fettreiches Netz, sowie die Gefahr eines Ileus durch Hindurchschlüpfen von abwärtigen Dünndarmschlingen durch den zuführenden Schenkel der Gastroenterostomieschlinge.

Diese Komplikationen treten nach Ansicht gewichtiger Autoren bei der vorderen Gastroenterostomie viel häufiger auf als nach der hinteren. Es steht in Frage, festzustellen, ob die Komplikationen tatsächlich so häufig sind und die eben erwähnte Annahme allgemein zu Recht besteht. Im folgenden gebe ich zunächst eine allgemeine Übersicht über unsere Fälle, zunächst die gutartigen Magenerkrankungen, dann die bösartigen und bespreche im Anschluß daran die Komplikationen, die auch wir im Anschluß an dic Gastroenterostomie erlebten.

Seit dem Jahre I90I sind in unserer Klinik noch 288 vordere Gastroenterostomien ausgeführt worden, und zwar I 53 bei gutartigen Erkrankungen des Magens, 135 bei inoperablen Carcinomen desselben. Nicht mitgezählt sind dabei die Gastroenterostomien, die als Teiloperation bei Magenresektionen (Billroth II. und I., Krönlein-Mikulicz wegen Ulcus und Carcinom) gemacht wurden, sondern nur diejenigen, die als selbständige Operation zur Umgehung des stenosierten Magenausganges vorgenommen wurden. Dagegen sind mitgezählt die Fälle von Ulcus duodeni und alle Gastroenterostomien, die wegen mechanischer Stenosen des Pylorus durch Adhäsionen usw. bedingt waren.

In bezug auf das Funktionieren der Gastroenterostomie habe ich mich über die Dauererfolge durch Versenden von Fragebogen an die Patienten zu orientieren versucht. Wenn ich auch nicht von allen habe Antwort erhalten können, so geht doch aus den mir zugegangenen Berichten hervor, daß die Erfolge gut waren. Zirkulationsstörungen, welche sich durch stärkeres Erbrechen gezeigt haben würden, sind in keinem Falle aufgetreten. Einige Patienten sind an interkurrenten Krankheiten gestorben. Die Überlebenden sind entweder ganz beschwerdefrei oder klagen nur 
über geringe Störungen, hauptsächlich nach reichlicherer Nahrungsaufnahme. Die Angabe von Schmerzen, die vielleicht auf das Entstehen eines neuen Geschwürs, insbesondere e in es peptischen Geschwürs des Dünndarms, hindeuten w ̈̈rde, fand sich in keinem der mir zugegangenen Beri chte. 2 Pat. kamen zur Wiederaufnahme wegen Verdachts auf Ulcus pepticum.

Naturgemä $\beta$ ist von Dauererfolgen bei den wegen Pylorusstenose auf Grund inoperabler Carcinome Gastroenterostomierten niche zu reden. Die Patienten sind meist an Kachexie und zunehmender Entkräftung oder an dem Hinzutreten einer interkurrenten Krankheit (Pneumonie) gestorben.

Was die genaueren Daten über die Resultate der vorderen Gastroenterostomie anbetrifft, so gehe ich .zunächst auf die bei den gutartigen Magenerkrankungen erzielten Erfolge ein. Sie gestalten sich folgendermaßen:

Von den I 53 Operierten wurden als geheilt oder wesentlich gebessert entlassen I 13, d. h. 73,20 Proz. In der Klinik gestorben sind 40 Operierte, d. i. 26, I3 Proz. Unter diesen starben:

I. An Pneumonie Pleuraempyem Pleuritis $12=7,8$ Proz. der Gesamtzahl der Operierten.

2. An Herzschwäche entweder infolge allgemeiner Körperschwäche oder star ker Anämie infolge Blutung aus dem Ulcus

3. An Peritonitis I $\mathrm{I}=7$, I Proz. der Operierten.

Unter diesen I I an Peritonitis nach Gastroenterostomie Gestorbenen sind 2 Fälle mitgezählt, die wegen Ulcus ventriculi perforatum in die Klinik eingeliefert wurden und die schon eine. Peritonitis hatten. Bei einem weiteren Patienten war die Bauchfellentzündung durch einen technischen Fehler (vergessener Tampon) hervorgerufen worden, bei 2 weiteren Patienten konnte bei der Obduktion eine bestimmte Ursache der Peritonitis nicht nachgewiesen werden.

4. An allgemeiner Sepsis nach ausgedehntem Decubitus bei außerordentlich starker Abmagerung

$$
\mathbf{r}=0,6 \text { Proz. der Operierten. }
$$


5. An postoperativem Tetanus (Katgut)

6. An Lungenembolie

$$
\mathrm{I}=0,6 \text { Proz. der Operierten. }
$$

$I=0,6$ Proz. der Operierten.

7. An lleus durch Abschnürung der untersten Ileumschlinge durch einen vom unteren Ende des großen Netzes nach abwärts ziehenden Adhäsionsstrang

$\mathrm{I}=0,6$ Proz. der Operierten.

8. An Circulus vitiosus $\quad \mathbf{2}=\mathbf{1}, 3$ Proz. der Operierten.

Bei dieser Übersicht muß, wie ich nochmals betonen möchte, in Betracht gezogen werden, daß sich unter den Verstorbenen eine Anzahl von Patienten findet, die entweder, wie bei den Ulcera, mit Blutungen, schon sehr stark ausgeblutet waren, oder wie bei den Fällen mit perforiertem Ulcus und schon bestehender Peritonitis von vornherein wenig oder keine Aussicht auf Heilung boten und wo die Operation nur als ein Versuch angesehen werden mußte; so stellt sich der Prozentsatz der geheilten oder gebesserten Fälle noch etwas höher (auf 80,5 Proz).

Wenn ich nun im folgenden auf die Komplikationen zu sprechen komme, welche auch wir bei den von uns wegen gutartiger Magenerkrankungen operierten Patienten erleben mußten, so erwähne ich zunächst den ersten von mir oben erwähnten Punkt, nämlich das Ulcus pepticum jejuni. Diese Komplikation konnten wir seit dem Jahre I9oI in zwei Fällen beobachten. Im ersten handelte es sich um einen Igjährigen Schnitter C. Dieser wurde wegen Ulcus ventriculi im Jahre I9I7 hier operiert (vordere Gastroenterostomie). Nachdem er zunächst nach der Entlassung ganz beschwerdefrei gewesen, stellten sich Ende 1918 wieder Schmerzen oberhalb des Nabels ein, worauf Patient wieder in die Klinik kam. Bei der erneut vorgenommenen. Operation wurde ein Ulcus pepticum jejuni, wie vermutet, festgestellt. Durch das Anlegen einer Braunschen Anastomose konnten die Beschwerden behoben werden. Patient wurde geheilt aus der Klinik entlassen. Abzuwarten bleibt natürlich, wie sich der weitere. Verlauf gestalten wird und ob Patient dauernd beschwerdefrei bleibt.

Im zweiten Fall (Sch.) war in Jahre I9I 5 eine vordere Gastroenterostomie angelegt worden. Ein Jahr später wurde Pat. mit Zeichen einer Ulcusperforation in die Klinik eingeliefert und kam 
zum Exitus. Die Obduktion ergab ein Ulcus pepticum an der Nahtstelle der Gastroenterostomie.

Unter den II 3 von uns als geheilt oder gebessert aus der Klinik entlassenen Fällen sind dieses, wie gesagt, die einzigen Fälle von Ulcus pepticum, die wir beobachten konnten. Das Ulcus pepticum stellt ja eine, wenn auch in letztet Zeit häufiger beschriebene, so doch immerhin verhältnismäßig seltene Erscheinung dar. Nachdem Brau ${ }^{1}$ ) im Jahre 1899 über den ersten Fall von Ulcus pepticum berichtete, konnten schon nach einem Jahre mehr ähnliche Beobachtungen gesammelt werden. $\mathrm{T}$ i e g el ${ }^{2}$ ) veröffentlichte 5 Ulcera peptica nach hinterer, 16 nach vorderer Gastroenterostomie, $\mathrm{Sch} \mathrm{os} \mathrm{ta} \mathrm{k}{ }^{3}$ ) 35 Fälle, unter denen 23 Ulcera nach vorderer, 9 nach hinterer Gastroenterostomie auftraten. Petré $n^{4}$ ) schilderte die Komplikation in 6, Schnitzler ${ }^{4}$ ) in ro Fällen. $\mathrm{Schwarz^{5 }}$ ) gab eine Statistik von 146 Fällen, davon $36 \mathrm{mit}$ tödlichem Ausgang. In dieser Statistik hält sich die Frequenz der Ulcera nach vorderer und hinterer Gastroenterostomie ungefähr die Wage. 1909 brachte Paterson $\left.{ }^{6}\right) 52$ Fälle und va $n$ Roojen ${ }^{7}$ ) 77 Fälle von Ulcus pepticum. In der Arbeit von Paterson sind eine ganze Reihe von Jejunalgeschwüren sowohl nach vorderer wie nach hinterer Gastroenterostomie aufgetreten. Die Feststellung des Zahlverhältnisses war mir hierbei wegen Unzugänglichkeit der Originalarbeit nicht möglich. In der Arbeit von van $\mathrm{R}$ oojen traten 29 Ulcera nach vorderer Gastroenterostomie, 12 nach derselben Operation mit Entero*

I) B ra u n, H. Verhandl. d. Deutschen Ges. f. Chir. 1899, 'Teil II.

2) T iegel, Uber peptische Geschwïre des Jejunums nach Gastro. enterostomie. Grenzgebiete Bd. I3.

3) Schostak, I. Das Ulcus pepticum jejuni und seine Bedeutung. Beiträge f. klin. Chir., Bd. 56, S. 360 .

4) Zitiert nach Keppler und Erkes, Zur Klinik des pylorusfernen Ulcus unter besonderer Berücksichtigung der Operationsresultate. Archiv f. klin. Chir. 1919, Bd. II I, H. 4, S.87!.

5) Schwarz, Beiträge zur. Kasuistik und chir. Therapie des peptischen Jejunalgeschwïrs. Archiv f. klin. Chir. I9I4, Bd. 104, S. 694.

6) Paterson, Jejunal and Gastrojejunal ulcer. Reprinted from the Proceed of the Roy. Soc. of Med., June 1909.

7) van $\mathrm{R}$ o o je $\mathrm{n}$, Uber das Ulcus pepticum nach Gastroenterostomie. Archiv f. klin. Chir. I909, Bd.9r, S. 38 r. 
anastomose, Io nach Gastroenterostomia ypsiliformis auf. Nach der Gastroenterostomia retrocolica posteriór wurden 22 Ulcera peptica beobachtet, nach der Gastroenterostomia retrocolica posterior ypsiliformis 3, mit Enteroąnastomose I Ulcus pepticum. Konnte v. Haberer schon im Jahre Igr 4 auf dem Chirurgenkongreß über 3 einschlägige Beobachtungen berichten, bei denen das peptische Geschwür übrigens alle dreimal nach hinterer Gastroenterostomie entstanden war, so konnte er im Jahre I9I9 in einer Arbeit ${ }^{1)} 13$ Fälle von Ulcus pepticum sammeln. Endlich ist im Juni 1919 eine sehr lesenswerte zusammenfassende Arbeit ${ }^{2}$ ) vom gleichen Autor erschienen, die über das Ulcus pepticum jejuni, darunter über 17 eigene Beobachtungen (durch Autopsie in operationem nachgewiesen), und zwar alle nach Gastroentero: stomia retrocol. post, , berichtet. Der Autor wendet sich auch gegen die Behauptung von $\mathrm{Sch}$ warz (1. c.), daß in bezug auf das Ulcus pepticum die Überlegenheit der hinteren Gastroenterostomie.über die anderen Methoden erwiesen sei und stützt sich dabei auf seine eigenen und die anderen, oben erwähnten Beobachtungen aus der Literatur. Weitere Mitteilungen über das Ulcus pepticum nach hinterer Gastroenterostomie finden sich bei Clairmont ${ }^{3}$, der über is Fälle aus der v. Eiselsbergschen Klinik verfügt, und bei Schmilinsky4). 2 Ulcera peptica wurden a ch in unserer Klinik nach hinterer Gastroenterostomie beobachtet.

Was die absolute Häufigkeit des Auftretens des Ulcus pepticum betrifft, so berechnet v. Haberer einen Prozentsatz von ca. 2: Ioo. Damit kongruiert auch die Statistik von v. Eisels berg, welcher auf 600 Gastroenterostomien 8 Ulcera peptica beobachtete. Vergleicht man damit die Ergebnisse der Statistik unserer Klinik, so finden sich gegenüber den erwähnten Zahlen

I) Haberer, v., Beiträge zu den auslösenden Ursachen des Ulcus pepticum jejuni post operationem. Wiener klin. Wochenschr. I9I9, Nr. I4.

2) $\mathrm{Hab}$ a rer, v., Das Ulcus pepticum jejuni, seine Erkennung und Behandlung. Zeitschr. f. ärztl. Fortbildung, Juni 1919, Nr. 12, S. 329. Wien 1913 .

3) Cla irmont. Verhandl. d. Ges. Deutscher Naturforscher u. Ärate,

4) Schmilinsky. Zentralbl, f. Chir. 1918, Nr, 25. 
keine wesentlichen Unterschiede (bei uns I,3 Proz., nach hinterer Gastroenterostomie bei uns in 5,4 Proz. der Fälle).

Überblickt man die oben erwähnten Ergebnisse aus den Mitteilungen der Literatur und die unserer eigenen Statistik, so läßt sich sagen, daß das Ulcus pepticum

I. verhältnismäßig selten ist und nur in $\mathbf{I}-2$ Proz. aller Fälle von Gastroenterostomie auftritt, und

2. bei alien Methoden der Gastroenierostomie in ziemlich gleicher Häufigkeit auftritt, woraus alsc weder der Vorzug der einen oder anderen Methode in bezug auf das peptische Jejunalgeschwür zu entnehmen ist. Die Ursache für die Annahme, daß das Ulcus pepticum jejuni hauptsächlich nach der vorderen Gastroenterostomie entstehe, ist darin zu suchen (worauf schon v. Haberer aufmerksam machte), daß früher bei fast ausschließlicher Anwendung der vorderen Gastroenterostomie das Ulcus pepticum natürlich nur bei dieser Methode beobachtet werden konnte; aber jetzt erst, wo die Gastroenterostomia post. retrocol. die weit häufiger angewendete Methode ist, mehren sich die Mitteilungen über auch in ihrem Anschluß auftretende Jejunalgeschwüre, so daß auf Grund obiger Referate aus der Literatur und unserer eigenen Beobachtungen eine ungefähr gleichmäßige Verteilung der Jejunalulcera auf die vordere und hintere Gastroenterostomie resultiert.

Muß man also bei allen Methoden der Gastroenterostomie mit der Entstehung des Ulcus pepticum rechnen, so ist unter Umständen das Auftreten eines solchen Geschwürs nach der Gastroenterostomia antecol. anterior noch günstiger, weil die Beseitigung desselben durch einen eventuellen neuen operativen Eingriff bei der vorderen Methode leichter ist, bei der hinteren aber in, manchen Fällen unmöglich sein kann, worauf schon Floercken 1 ) aufmerksam machte.

Ob alle Ulcera peptica, die beschrieben sind, tatsächlich als solche im wahren Sinne anzusehen sind, bleibt dahingestellt. Möglicherweise läßt sich aber die Zahl der wahren Ulcera peptica noch verkleinern, wenn man alle diejenigen ausschaltet, die ihren Ausgangspunkt von der Schleimhautnahtlinie der Gastroentero-

I) Floercken, Zur Behandlung des Ulcus pepticum jejuni nach Gastroenterostomie. Zentralbl. f. Chir. 1918, Nr. 33, S. 568. 
stomie nehmen, für deren Entstehen oft mangelhaftes Nahtmaterial oder ein kleiner technischer Fehler, der sich im Einzelfalle nicht immer wird nachweisen lassen, verantwortlich gemacht werden könnte. Ist es doch nicht verwunderlich, wenn ein Ulcus an der Nahtlinie entsteht, wenn der Faden nicht gehalten oder nicht fest genug angezogen worden ist, die Schleimhautränder etwas auseinander wichen und so ein locus minoris resistentiae für die Einwirkung des Magensaftes gegeben ist. Werden sich solche Zufälle nicht immer vermeiden lassen, so dürfen andererseits die durch sie entstehenden Geschwüre nicht mit in die Statistik der wahren Ulcera jejuni hineingerechnet werden, wie das vielleicht für die Fälle von Tiegel (1. c.), Lieble in und Hilgenreiner ${ }^{1}$ ) zutreffen dürte, bei denen die Geschwüre am Gastroenterostomiering lokalisiert waren. Um solche Nahtliniengeschwüre zu vermeiden, sollte deshalb keine Seide, sondern ausschließlich Katgut - wie schon von mancher anderen Seite hervorgehoben worden ist - zur Schleimhautnaht Verwendung finden.

Zwar will v. Haberer technische Fehler bei der Entstehung der Ulcera ausschalten können, was natürlich für die Operateure mit viel Erfahrung zutrifft; aber es werden doch viele Gastroenterostomien auch von jüngeren Operateuren ausgeführt, wobei dann ein oder das andere technische Moment wohl einmal nicht so genügend beachtet wird.

Mag die technische Frage weniger vielleicht als das Nahtmaterial bei Entstehung des Ulcus pepticum an der Nahtstelle ins Gewicht fallen, so spielt sie doch eine größere Rolle bei der zweiten wichtigen Komplikation der Gastroenterostomie, den Passagestörungen, die wir als Circulus vitiosus zusammenzufassen gewöhnt sind. Von solchen Störungen erlebten wir bei den 153 voln uns operierten gutartigen Magenerkrankungen bzw. Pylorusstenosen 5, von denen 3 geheilt wurden, 2 gestorben sind; unter den 135 wegen inoperablen Carcinoms Gastroenterostomierten. waren es 5, Fälle, die alle 5 gestorben sind. $O b$ alle diese Fälle

1) Li e b le in und $\mathrm{H}$ ilgenreiner, Das Ulcus pepticum jejuni nach Gastroenterostomie, Deutsche Zeitschr. I. Chir. 1905, Bd. 21. Siehe auch bei v. H a berer. Zeitschr. f. ärztl. Fortbildung 19I9, Nr. r2, S. 335. 
als echte "Circulus vitiosi“" anzusehen sind, und auf ihre Beurteilung darf ich weiter unten kurz eingehen, lasse aber zuvor ein paar kurze Auszüge aus den Krankengeschichten folgen.

I. Passagestörungen bei den von uns operierten gutartigen Magenerkrankungen bzw. Pylorusstenosen.

Fall I: E. Sch., Arbeiterfrau, 32 Jahre alt. In der Klinik vom 28. XI. I9I4 bis 20. I. I9I5. A nam n e se: Vor 9 Jahren intern wegen Magengeschwürs behandelt. In letzter Zeit Blut im Stuhl, häufiges Erbrechen, Schmerzen nach dem Essen.

$\mathrm{S}$ t a $\mathrm{t}$ u s: Unter dem rechten Rippenbogen dicht bei der MedianJinic lcbhafter Druckschmerz. Gleichfalls Druckschmerz rechts yom Nabel. Röntgenol. mittelgroßer 6-Stunden-Rest; I Querfinger oberhalb davon r. neben der Wirbelsäule nischenförmiger Schatten (Duodenalfleck). Lebhafte Peristaltik.

Diagnose: Ulcus juxta-pyloricum.

Operation: In der Gegend des Pylorus breite Adhäsionen. Am absteigenden Ast des. Duodenums gleichfalls viel Adhäsionen, wodurch dieser ganz nach dem Pylorus und dem oberen Ast des Duodenums herangezogen erscheint. Ulcus nicht deutlich palpabel: Ausschaltung des Pylorus durch Umschnürung mit dünnem Jodoformgazedocht. Typische Gastroenterostomia antecolica anterior mit Aufhängen beider Schenkel der Gastroenterostomieschlinge nach Kappeler.

Verla uf: Primäre Wundheilung. 30.XII. 1914. Zunehmende Schmerzen und lebhaftes Erbrechen. Es wird Abknickung des abführenden Schenkels der Gastroenterostomie angenommen und nochmals laparotomiert. Der abführende Schenkel ist kollabiert, spitzwinklig abgeknickt, nach dem oberien $\mathrm{W}$ undwinkel herangezogen und dort fixiert. Die Adhäsionen werden gelöst. Zwischen dem zuführenden gefüllten Schenkel der Gastroenterostomie und dem abführenden Schenkel Anlegen einer Braunschen Enteroanastomose. PrimäreWundheilung. 20. I. 1915. Geheilt und beschwerdefrei entlassen. Röntgenologisch : Entleerung durch die Gastroenteroanastomose gut. Ein Circulus vitiosus im wahren Sinne des Wortes hatte also nicht bestanden.

Fall 2. Sch. B., 46 Jahre, Arbeiterfrau. A n a mn e se, die für Ulcus am Pylorus spricht.

St a tu s: Röntgenol. 6-Stunden-Rest. Eine schmale, geschlängelte Wismutstraße zieht von hier aus aufwärts nach der Gegend der etwa in Nabelhöhe befindlichen Narbe am rechten Rektusrand (Appendektomienarbe). An dieser Stelle ein trichterförmiger Wismutschatten. 
D i a g n o s e: Vielleicht Ulcus mit Narbenverziehungen, vielleicht Adhäsionen zwischen Pylorus und der alten Laparotomienarbe.

Operation 25.III. 1913: Abknickung des Pylorus durch breite, sehr zahlreiche Adhäsionen. Duodenum gleichfalls durch Adhäsionen verzogen. Gastroenterostomia ant. ant. mit Kappelerscher.Aufhängung der zu- und abführenden Schlinge. Um das Colon transvers. zum Aufsuchen der Jejunumschlinge heraufklappen zu können, müssen erst Verwachsungen des großen Netzes nach dem kleinen Becken hin gelöst werden. Jejunumschlinge, um Störungen durch das verwachsene Netz zu vermeiden, $60 \mathrm{~cm}$ lang gewählt.

Verla uf: Zunächst glatter Verlauf, dann (8. IV. 1913) heftige Leibschmerzen. Röntgenol. Stagnation. Entleerung durch die Gastroenterostomie nur, wenn der Magen eine bestimmte Füllung erreicht hat und gewissermaßen überläuft. Zunehmende, sehr heftige Schmerzen, keine Darmsteifung. Verdacht auf Circulus vitiosus. Relaparotomie: Uberall haben sich wieder sehr zahlreiche und breite Adhäsionen gebildet. Zuführender Schenkel gebläht, seine Wand hypertrophiert und mit dem abführenden Schenkel, wie zur Vorbereitung: der Braunschen Anastomose, durch frische Verklebungen verbunden. Ausführung derselben. Der bis ins kleine Becken herunterhängende Magen wird an der vorderen Bauchwand und dem Ligamentum teres hepat. mit einigen Nähten fixiert. Normaler Verlauf. Keine Schmerzen, kein Erbrechen. Geheilt entlassen.

F a 11 3. H. K., 42 Jahre, Landsturmmann. In der Klinik vom 19. IX. I9I6 bis 3. I. I917. A namnese: Seit 1o Jahren zeitweise Auftreten von Schmerzen in der Magengegend, die nach der Nahrungsaufnahme aufhören. Während der Beobachtungszeit auf der inneren Klinik zunehmendes Erbrechen.

St a tus: Ausgesprochene Plätschergeräusche in der Oberbauchgegend. Ausgesprochene Stagnation im Magen. Röntgenol.: Ausgesprochener 6-Stunden-Rest. Tiefstand des Magens bis ins kleine Becken. Pylorusgegend auf Druck sehr schmerzhaft.

Di a g nos e: Pylorusstenose, wahrscheinlich durch Ulcusnarbe.

Operation 22. IX.: Strahlige Narbe am Pylorus; dieser vollkommen verschlossen. Gastroenterostomia ant. ant. ohne Braunsche Anastomose, o hne Kappelersche Aufhängung.

Vèrla uf: Aseptischer Verlauf. Patient will sich jedoch nicht recht erholen. Zeitweise Erbrechen. 25.X. Starke Magenblähung. Leib in seiner oberen Hälfte stark aufgetrieben. II. XI. Heftiges Erbrechen. Relaparotomie: Breite Verwachsungen an der Gastroenterostomiestelle, dic flächenhaft auf den ganzen Magen übergreifen. Der zuführende Schenkel der Gastroenterostomie stark hypertrophisch, anscheinend hat ein Arbeiten gegen einen Widerstand in verkehrter Richtung stattgefunden. Resektion der Gastroenterostomiestelle mit dem entsprechenden Magenabschnitt. Heilung. 
Fall 4. B. V., 46 Jahre. 8. X. bis 19. X. I9I4. Gestorben. Ana$m$ nese: Schmerzen in der Nabelgegend, starkes Erbrechen nach dem Essen. Aufstoßen. Starke Gewichtsabnahme.

Dia g nose:-Ulcusnarbe am Pylorus.

Operation 12.X.: Beim Offnen des Peritoneums sehr reichlich Adhäsionen zwischen Magen, Duodenum, Quercolon und Netz. Sie umgeben den Magen wie mit einer Hülle. Am Pylorus alte Ulcusarbe. Lösung der Adhäsionen. Gastroenterostomia ant. ant. Aufhängen der Schlinge nach Kappeler.

Verlauf: In den ersten Tagen nach der Operation heftiges Erbrechen. Deshalb am I9.X. nochmalige Laparotomie. Zuführende Schlinge der Gastroenterostomie gebläht, abführende kollabiert. Braunsche Anastomose. Zunehmende Herzschwäche. Exitus.

Obduktion: Status nach Gastroenterostomie. Gastroenterostomieschlinge $\mathrm{I} \mathrm{m}$. unterhalb der Flexura duodeno-jejunalis. Circulus vitiosus. Chron. adhäsive Peritonitis. Ulcusnarbe am Pylorus.

Fall 5. G. W., 49 Jahre. 16. VII. bis I3. VIII. 1918. Gestorben. Anamnese: 1917 wegen Ulcus ventriculi in der Klinik zur Beobachtung. Mit Diätvorschriften entlassen. 1918 wieder starke Schmerzen in der Magengegend. Hungerschmerz. Schmerzen I Stunde nach dem Essen. Kein Erbrechen.

Status: Duodenalgegend druckempfindlich. Blut im Stuhl. Mäßige Säurereste im Mageninhalt.

Röntgenol.: Keine Aussparung, kein 6-Stunden-Rest.

Diagnose: Ulcus duodeni. Am 18. VII. Gastroenterostomia ant. ant. Diagnose eines Ulcus duodeni bestätigt. Perigastritis.

Verlauf: Nach 6 Tagen heftiges Erbrechen. Keine Speisen werden im Magen behalten. Offenbar Circulus vitiosus. Deswegen Laparotomie. Blähung und starke Füllung des zuführenden Schenkels, Kollaps des abführenden. Ausgedehnte Adhäsionen an der Gastroenterostomiestelle. Braunsche Anastomose. Auch .durch diese wird der Circulus vitiosus nicht behoben. Zunehmende Schwäche des Pat. Er verlangt selbst eine erneute Operation. Es wird beschlossen; Gastroenterostomieschlinge und den entsprechenden Magenabschnitt zu resezieren. Dieses gelingt unter Schwierigkeiten. Bildung eincs Duodenalstumpfes. Verbindung des oralen Magenstumpfes mit den abführenden Schenkel der Gastroenterostomie und Einnähen des zuführenden Schenkels in den abführenden End-zu-Seit. - Pat. übersteht den Eingriff nicht. Zunehmende Herzschwäche. Exitus. Eine Ursache des Circulus vitiosus wurde nicht gefunden. Keine Spornbildung. Vielleicht Magenatonie. 
II. Passagestörungen in Fällen von Gastroenterostomia antecolica anterior wegen inoperablen Carcinoms des Magens.

Von den 135 wegen inoperablen Carcinoms Gastroenterostomierten wurden $74=54,8 \mathrm{I}$ Proz., meist gebessert, aus der Klinik entlassen. In der Klinik starben $6 \mathrm{I}=45$, I 8 Proz. Unter diesen starben

I. an Pneumonie oder

Pleuritis

2. an Lungenembolie $18=13,33$ Proz. der Operielten,

3. an Kachexie und Herzschwäche entweder bald nach der Operation oder im Laufe der nächsten 8-i 4 Tage

4. an Peritonitis durch Nahtinsuffizienz

5. an Peritonitis nach Hernieneinklemmung

6. an Peritonitis durch Per-

$$
28=20,73 \quad, \quad, \quad, \quad,,
$$

$$
6=4,44,, \quad, \quad,
$$$$
\mathrm{I}=0,73 \quad ; \quad, \cdots \quad,
$$
foration des Tumors in die freie Bauchhöhle

7. an Passagestörungen

8. an Ileus

$$
\begin{array}{llll}
I=0,73 & , & , & , \\
5=3,33 & , & , & , \\
I=0,73 & , & , & ,
\end{array}
$$

Die aus der Klinik zunächst gebessert Entlassenen sind alle gestorben, entweder an interkurrenten Krankheiten oder an Kachexie I Tag bis I Jahr nach der Entlassung.

Auf Punkt 7 - die Passagestörungen - darf ich nun etwas näher eingehen und im folgenden zunächst die Krankengeschich: ten kurz wiedergeben.

Fall I. P. J., 46 Jahre, Maurer. In der Klinik vom 5. VI. bis 18.VI. 1902. Typische Anamnese für Carcinoma ventriculi. Grobe Abmagerung. Palpabler Tumor. Pylorusstenose.

Operation 9.VI.: Tumor inoperabel. Wegen der Stenosenerscheinungen Gastroenterostomia ant. ant. Aufhängung der Gastroenterostomieschlinge nach Kappeler. Zunächst normaler Verlauf. Dann Temperaturanstieg mit kolikartigen Leibschmerzen, Auftreibung des Leibes. Kein Erbrechen. Verfall. Exitus.

Obduktion: Sie klärt die Erscheinungen vollkommen. Die 
Jeujnumschlinge war $10 \mathrm{~cm}$ von der Plica duodeno-jejunalis entfernt anisoperistaltis ch an dem Fundus des Magens angenäht. Nahtstelle intakt. Beim Auflegen der Schlinge auf den Magen war der zuführende Schenkel $90 \mathrm{bzw}$. I80 Grad gedreht, so daß ein völliger Verschluß desselben etwas unterhalb des Ubergangs vom Duodenums ins Jejunum zustande gekommen war. Durch Druckgangrän war eine Perforation an der Drehungsstelle eingetreten und Peri. tonitis entstanden.

F all 2. E. K., 50 Jahre, Werkführer. In der Klinik vom 3r.X. bis 17 . XI. Igo6. Typische Anamnese für Carcinoma ventriculi. Pylorusstenose. Starke Kachexie. Palpabler Tumor.

Operation 3. XI.: Starke Ptose und Dilatation des Magens. Am Pylorus harter, hühnereigroßer Tumor. Drüsenmetastasen. Deshalb $G$ astroenterostomia ant. ant. Kappelersche Aufhängung der Schenkel der Gastroenterostomieschlinge. Verlauf anfangs ohne Beschwerden, dann heftiges Erbrechen, das in den nächsten Tagen, fäkulent wird und trotz Magenspülungen nicht zu stillen ist. 17. XI. Exitus.

Obduktion: Gastroenterostomienaht tadellos. Zuführender Schenkel der Gastroenterostomieschlinge nur $22 \mathrm{~cm}$ lang. Duodenum weit. Sonst ist der Darm kontrahiert, besonders clas Colon. Offenbar hat Circulus vitiosus als Folge der zu kurzen zuführenden Schlinge bestanden.

Fall 3. J. O., 49 Jahre, Tapeziersfrau. 30. III. bis 21. IV. 1903 in der Klinik. Anamnese, die für maligne Pylorusstenose spricht. Palpable Resistenz oberhalb des Nabels. Laparotomie 2. VI. Dilatierter, atonischer, ptotischer Magen. Am Pylorus hühnereigroßes Carcinom, vollkommen stenosierend. Netz in der Umgebung carcinomatös. Gastroenterostomia ant. ant. mit $30 \mathrm{~cm}$ langer Schlinge und Kappelerscher Aufhängung derselben. Verlauf zunächst unge-stört, dann zunehmendes Erbrechen. Verfall. Exitus 21. IV.

Obduktion: Offnung nach dem abführenden Darm durch S pornbildung geschlossen, zuführender Schenkel weit.

Fall 4. W. F., 54 Jahre, Roßschlächter. In der Klinik vom 3. II. bis 21.II. 1903. Starke Gewichtsabnahme. Magenbeschwerden allgemeiner Art. Tumor in der Magengegend deutlich palpabel.

Operation 4. II.: Carcinom. Tumor am Pylorus, allseitig verwachsen. Gastroenterostomia ant. ant. mit Kappelerscher Aufhängung. der Gastroenterostomieschenkel. Verlauf zunächst glatt, dann zunehmendes Erbrechen. 17. II. Relaparotomie. Keine Zeichen für Circulus vitiosus. Magen stark dilatiert. Patient erholt sich nicht mehr. Exitus 31. II. 1903.

Obduktion: Keine sicheren Anhaltspunkte für Circulus vitiosus. Ab- und zuführender Schenkel gleich weit, keine Spornbildung. 
Gastroenterostomiestelle intakt. Keine Abknickung. Magen sehr dilatiert.

Fall 5. G. R., 44 Jahre, Tischler. In der Klinik vom I2. IX. bis 23. IX. I9I9. Typische Anamnese für 'bösartigen Tumor des $\mathrm{Ma}$ gens mit Pylorusstenose.

Operation 17.IX.: Inoperables Carcinom des Magens mit Metastasen. Gastroenterostomia ant. ant. mit Kappelerscher Aufhängung der Gastroenterostomieschenkel. Verlauf zunächst gut. Seit 20. IX. zunehmendes Erbrechen. 23. IX. Exitus.

Obduktion ergibt einen engen abführenden und einen ctwas weiteren zuführenden Schenkel der Gastroenteröstomieschlinge. Dünnund Dickdarm kollabiert. Ursache des Circulus witiosus, der wahrscheinlich vorhanden gewesen, nicht aufgeklärt, vielleicht Atonie des Magens.

Wenn wir die eben im Auszug erwähnten Krankengeschichten bzw. die in ihnen verzeichneten Zirkulationsstörungen richtig beurteilen wollen, dann darf es erlaubt sein, zunächst den Begriff des Circulus vitiosus näher zu definieren. Denn je nachdem, wie man den Begriff des Circulus vitiosus faßt, hängt es $a b$, welche Fälle von Passagestörungen im Gebiet der Gastroenterostomiestelle man in eine aufzustellende' Statistik hineinrechnet. Bekanntlich ist der Begriff des Circulus vitiosus von v. Mikulicz ${ }^{1}$ ) folgendermaßen definiert worden. Auf dem Chirurgenkongre $B$ I897 sagt er: Beim Circulus vitiosus ,entleert sich der Mageninhalt nicht, wie gewünscht wird, in das abführende Darmstück, sondern in den zuführenden Darmschenkel, ins Duodenum; der zuführende Darm regurgitiert seinen Inhalt wieder in den Magen. ... Bei der Sektion findet man den zuführenden Schenkel des Jejunums kolossal ausgedehnt, der abführende Schenkel ist leer, zwischen dem zu- und abführenden bildet sich eine Art Sporn ..." Wenn wir von dieser Definition ausgehen wollten und nur die Fälle, die wir bei unseren Gastroenterostomierten durch echte Spornbildung erlebten, in unsere Statistik hineinrechnen wollten, so brauchten wir bloB einen Fall (Fall II, 3) anzuführen. Wenn wir aber, wie im folgenden, den Wert einer Methode prüfen wollten, so mußten wir, wie aus den vorstehenden Kranken-

I) M ikulicz, v., Die chir. Behandlung des chron. Magengeschwürs. Verhandl. d. Deutschen Ges. f. Chir. 1897, 26. KongreB. Zentralbl. f:'Chir. $1897, \mathrm{Nir} 28, \mathrm{~S} .69$. 
geschichten zu ersehen ist, hier den Begriff des Circulus vitiosus weiter fassen und alle Passagestörungen im Gebiet der Gastroenterostomieschlinge anführen, die vielleicht auf die Art der Methode der Gastroenterostomie hätten zurückgeführt werden können, also alle Fälle, die auch klinisch die Anzeichen des Circulus boten.

Gehen wir also im einzelnen auf unsere Fälle ein, so scheiden 3 Fälle von vornherein aus, die durch nachgewiesene technische Fehler zustande kamen (Fall I/3, II/I und II/2). In einem Fall war die Kappelersche Aufhängung vergessen worden, die Schenkel der Gastroenterostomieschlinge hingen schlaff und parallel zueinander herab, und die Vorbedingung zum Einfließen des Mageninhalts in den zuführenden Schenkel war von vornherein gegeben. Begünstigt war die Komplikation noch durch die zahlreichen breiten, flächenhaften Adhäsionen, in die die ganze Gastroenterostomiestelle eingehüllt war, und die so eine spontane Richtigstellung der Schenkel verhinderten. Trotzdem war ein Sporn nicht entstanden. Im zweiten Fall (Fall I//I) war die Gastroenterostomieschlinge antiperistaltis $\mathrm{ch}$ gelagert und viel zu kurz genommen worden ( $\mathrm{IO} \mathrm{cm}$ ). Im dritten Fall (Fall II/2) ist gleichfalls eine zu kurze Schlinge zur Gastroenterostomie verwendet worden. Außerdem hat sicher die motorische Insuffizienz des Magens, wie die Ptose und Dilatation, zur Entstehung der Passagestörung beigetragen. Diese 3 Fälle hätten, die Vermeidung der technischen Fehler vorausgesetzt, mit größter Wahrscheinlichkeit einen guten Verlauf genommen, bzw. es hätte eine zweite Operation vermieden werden können.

In Fall I/I war die Zirkulationsstörung durch Abknickung des abführenden Gastroenterostomieschenkels infolge Adhärenz im oberen Winkel der Bauchdeckenwunde zustande gekommen, wiederum begünstigt durch die Tendenz des Peritoneums, Adhäsionen zu bilden. Eine ähnliche Beobachtung machte La mp ${ }^{1}$ ), indem die Zirkulationsstörung gleichfalls durch Adhärenz der Anastomose an der vorderen Bauchwand zustande kam, aber durch' B ra u nche Enteroanastomose behoben werden konnte. Lampe

I) L a mpe. Die Funktionsstörungen der Wölfler-Lückeschen Gastroanastomose innerhalb des I. und 2. Tages. Verhandl. d. Deutschen Ges. f. Chir. 1904, 33. KongreB, S. 93. 
empfiehlt in ähnlichen Fällen, wo durch den Befund Komplikationen im Verlauf zu erwarten sind, von vornherein eine Bra unsche Anastomose auszuführen oder ein "Anastomosenrohr" einzuiegen. So kann in diesem Fall, in dem die Passagestörung nicht als echter Circulus vitiosus anzusehen ist, sondern durch andere Nebenumstände'entstanden war, die Methode der Gastroenterostomie nicht für den zunächst schlechten Verlauf verantwortlich gemacht werden.

In Fall $\mathrm{l} / 2$ waren gleichfalls schon bei Eröffnung des thdomens zahlreiche Verwachsungen vorhanden und hatten sich dann, wie aus dem Befunde der Relaparotomie ersichtlich, noch stark vermehrt. Trotz Kappelscher Aufhängung lagen die Schenkel der Gastroenterostomieschlinge parallel und vertikal nach abwärts und waren bereits, wie als Vorbereitung zur Bra un schen Anastomose" miteinander verklebt. An dieser schnellen und festen Verklebung der beiden Schenkel sind sicher die Adhäsionen mit schuld. Weiter dürfte auch die im Anschluß an dic Operation aufgetretene Atonie und starke Dilatation des Magens zur Entstehung der Zirkulationsstörung beigetragen haben.

Ähnlich lagen die Verhạltnisse in Fall $\mathrm{I} / 4$. Wodurch der Circulus vitiosus in Fall $\mathrm{I} / 5$ bedingt war, hat sich nicht sicher entscheiden lassen. Spornbildung war nicht vorhanden. Jedenfalls hat Atonie des Magens eine erhebliche Rolle gespielt.

Sicher wird von mancher Seite der Einwurf erhoben werden, daß diese Adhäsionen, wie in den letzten beiden Fällen, durch die hintere Gastroenterostomie vermieden worden wären. Dagegen muß ich erwähnen, daß gerade bei der hinteren Gastroenterostomie die Bildung von ausgedehnten Adhäsionen und in ihrem Gefolge Abknickung der abführenden Schlinge beobachtet worden ist (Kocher $\left.{ }^{1}\right)$ ). Vergegenwärtigt man sich. solche Fälle, in denen die Zirkulationsstörung auf Adhäsionsbildung zurückzuführen ist, so erscheint die Beseitigung der nach der Gastro enterostomie auftretenden Zufälle, wenn eine hintere Gastroenterostomie ausgeführt ist, besonders schwierig, und das je mehr, je kürzer die zur Gastroenterostomie verwandte Jejunumschlinge ge-

I) Kocher, Ih., Die chirurgische Therapie der Magenleiden. Grenzgebiete Bd. 20, S. 891 . 
nommen wird. So wird bei der hinteren Gastroenterostomie unter Umständen das Anlegen einer Enteroanastomose bei ganz kurzer Schlinge unmöglich und, wie aus Belegen aus der Literatur ersichtlich, die Ausführung einer vorderen Gastroenterostomie zur Beseitigung der Passagestörung infolge hinterer Gastroenterostomie nötig. Eine solche Beobachtung veröffentlichte Vullièt ${ }^{1}$ ), indem der Circulus vitiosus, nach hinterer Gastroenterostomie entstanden, durch vordere Gastroenterostomie behoben werden mußte. Eine zweite Beobachtung finde ich bei Tanberg ${ }^{2}$ ), der 2 Fälle von Circulus nach hinterer Gastroenterostomie veröffentlichte, von denen der erste durch vordere Gastroenterostomie mit Braunscher Anastomose, der zweite erst nach einem vergeblichen Versuch mit vorderer Anastomose durch Resectio pylori et jejuni und Rouxsche Operation geheilt werden konnte. Schließlich finde ich noch .2 Fälle von Circulus vitiosus nach hinterer Gastroenterostomie bei $\mathrm{Dubs}^{3}$ ), bei denen trotz Wahl der kürzesten Jejunumschlinge (nach der Forderung von Czerny, Bier, Hochenegg, Porges, Petersen) die Zirkulationsstörung entstanden war, ein leider zu später Versuch mit vorderer Gastroenterostomie zu ihrer Behebung aber keine Heilung brachte. Beide Fälle sollen durch hochgradige Ptose und Dilatation, sowie motorische Insuffizienz (Atonie) des Magens entstanden sein. Der sich immer mehr füllende Magen hatte dann eine Kompression des abführenden Schenkels der Gastroenterostomieschlinge herbeigeführt. Dubs stellt am Ende seiner Arbeit den Grundsatz auf, daß da, wo die genannten Momente im Zustande des Magens zusammentreffen, eine hintere Gastroenterostomie nicht angelegt werden darf, sondern entweder nach $F_{\text {a }}$ us $c h$ eine Jejunostomie oder eine vordere Gastroenterostomie bzw. Magen-Pylorusresektion ausgeführt werden soll.

I) Vulliet, H., Le "Circulus vitiosus" après la gastroentérostomie et les moyens d'y remédier. La mobilisation de l'anse duodeno-jejunale. Revue med. de la. Suisse Romande XXXVIII, annéc 1918, No. 12. Ref. Zentralbl. f. Chir. 1919, Nr. 29, S. 574.

2) Ta nberg, O., Ein Beitrag zur Frage der Kombinationen nach Gastroenterostomien. Norsk. Magazin for Laegvidenskaben, 78. Jahrg., Nr. 3, S. 325. Ref. Zentralbl. f. Chir. 1918, Nr. 35, S. 623.

3) Dubs, J. "Circulus" nach hinterer Gastroenterostomie. Zentralbl. f. Chir. 19I9, Nr.12, S. 21 I. 
Es muß mehr oder weniger als Zufall angesehen werden, daß die Adhäsionen sich in den oben erwähnten Fällen gerade im Gebiet der Gastroenterostomieschlinge entwickelt haben und dadurch die Erscheinungen des Circulus entstanden; gerade ebenso hätte es geschehen können, đaß sich Adhäsionen an anderen Stellen des Abdomens entwickelt und Abschnürung, Abklemmung oder Abknickung des Darmes bewirkt hätten, wie man das ja öfters nach Bauchoperationen sieht. Auf keinen Fall kann die angewendete Methode für die eingetretene Komplikation verantwortlich gemacht werden.

Von unseren Fällen bleiben noch 4 übrig (Fall I/5, II/3, II/4, II/5). Der erste entstand durch Spornbildung, die sich trotz Kappelerscher Aufhängung entwickelt hatte. Die drei anderen ergaben keine pathologisch-anatomische Ursache der Zirkulationsstörungen. Vermutungsweise wurde vom patholog. Anatomen Atonie des Magens angenommen. Es hat sich also in diesen Fällen lediglich um einen klinischen Circulus vitiosus gehandelt. Sie schließen sich den beiden Fällen von $\mathrm{Dubs}$ (1. c.) an, zumal (wenigstens in zwei) starke, Ptosis und Dilatation des Magens auch in unseren Fällen vorhanden waren. In der dritten Krankengeschichte habe ich ähnliche Angaben nicht finden können; doch muß eine motorische Insuffizienz auch hier in Ermangelung anatomischer Grundlagen angenommen werden. Ähnlich den Beobachtungen von $\mathrm{Dub}$.s sind die von $\mathrm{Kausch}$ ) aus dem Jahre 1916. Nur möchte ich besonders bemerken, daß diese Fälle alle nach $h$ interer Gastroenterostomie entstanden, während unsere Fälle nach vorderer Gastroenterostomie auftraten.

Es hat bisher, wie schon oben erwähnt, die Ansicht bestanden, daß Störungen im Sinne des Circulus vitiosus nach hinterer Gastroenterostomie fast überhaupt nicht auftreten. Diese Auffassung kommt nicht nur in der Arbeit von Dubs zum Ausdruck, sondern ist auch von Bier (1. c. S. I I3) in dem Satz festgelegt worden: „Die hintere Gastroenterostomie ... hat vor der vorderen ganz erhebliche Vorteile. Die gefürchtetste Komplikation, der Circulus vitiosus, scheint bei ihr überhaupt nicht vorzukommen." Diese Auffassung dürte bis zu einem gewissen Grade richtig

I) Kausch, W., Der Circulus vitiosus nach der Gastroenterostomie. Deutsche med. Wochenschr. 1916, Nr.28, S. $845-847$. 
sein, wenn man den Begriff des Circulus mit v. Mikulicz verhältnismäßig eng faßt und seine Entstehung auf die Spornbildung allein zurückführt. Aber auch in diesem Sinne ist die Annahme eben nur bis zu einem gewissen Grade richtig; denn es sind Fälle von pathologisch-anatomisch nachgewiesener Spornbildung auch bei hinterer Gastroenterostomie festgestellt worden. 3 solche Fälle veröffentlichte Föderli) im Jahre 1903. Faßt man den Begriff des Circulus vitiosus aber weiter in dem Sinne, wie ich es oben kurz dargelegt habe, so lassen sich noch zahlreiche andere Fälle von Passagestörungen nach hinterer Gastroenterostomie aus der Literatur anführen. Ich erwähne sie der Vollständigkeit halber kurz nacheinander. Noetze $1^{2}$ ) berichtet über einen 7 Tage nach hinterer Gastroenterostomie entstandenen stürmischen Circulus vitiosus, bei dem zuerst eine Gastrostomie nach $\mathrm{W}$ itzel vorgenommen wurde. Da die Lösung des Circulus aber nicht erfolgte, mußte, da eine Anastomose nach $\mathrm{Braun}$ wegen zu kurzer zuführender Schlinge nicht möglich war, eine Anastomose zwischen Jejunum und Pars inferior duod. ausgeführt werden, die den gewünschten Erfolg brachte. N oe tzel' schließt damit, daß die unangenehme Komplikation durch keine Methode ganz vermieden würde.

2 Fälle von Circulus nach hinterer Gastroenterostomie finde ich bei $\mathrm{Mauclair} \mathrm{e}^{3}$ ), die durch „Knieellenbogenlagerung“ geheilt wurden. Welcher Art die Passagestörung war, läßt sich deshalb nicht genau feststellen. S $\mathrm{r}$ ä $\mathrm{te}^{4}$ ) befaßt sich in einer längeren Arbeit mit den verschiedenen Arten der Unwegsamkeit des Verdauungstraktus nach Gastroenterostomie und empfiehlt die v. Hackersche Methode, berichtet aber gleichzeitig über I I Circulus-vitiosus-Fälle, die er im Anschluß an mit dieser Me-

I) Föd e r 1, O., Über den Circulus vitiosus nach Gastroenterostomie. Wiener klin. Wochenschr. I903, Nr. 4I.

2) Noetzel, Diskussion zu dem Vortrag von Kocher: Über Ulcus ventriculi und Gastroenterostomie. Verhandl. d. Deutschen Ges. f. Chir. I9I2, 4I. Kongreß, S. 48.

3) M a ucla ire, Deux cas de circulus vitiosus traités par position genu-pectorale. Arch. génér. de chir. I9ı2, VI, 6. Ref. Zentralbl. f. Chir. 1912, Bd. 2, Nr. 37, S. 1277.

4) Sträter, M. E., Darmocclussie en Gastroenterostomia. Amsterdam, J. Clausen, 1899,85 S. Ref. Zentralbl. f. Chir. I899, I, S. 245. 
thode operierte Fälle erlebte 4 Proz.). Hercherl) beobachtete eincn Fall von Circulus nach hinterer Gastroenterostomie, der scheinbar durch Ptose und Dilatation des Magens zustande kam. Beseitigt wurde der Zustand durch eine Gastropexie nach Rovsing. Ähnliche Fälle von Circulus vitiosus finden sich bei $\mathrm{Mad}$ sen $\%$, bei $\mathrm{Schwarz}{ }^{3}$ ) (ohne Zahlenangabe) und bei Dobrotworski ${ }^{4}$, der in einer genauen Statistik über die westeuropäischen und russischen Fälle von Gastroenterostomie 23 Fälle von Circulus vitiosus nach hinterer, 56 nach vorderer Gastroenterostomie und vereinzelte Fälle nach einigen anderen Modifikationen der Gastroenterostomie anführt. Dobrotworski stützt sich dabei auf 5i26 Fälle von Gastroenterostómien.

In einer Arbeit über die Gastroenterostomie berichtet Le roy über die von $\mathrm{Hartm}$ a $\mathrm{n}$ ausgeführten Gastroenterostomien und will im Anschluß an die Gastroenterostomia retrocolica keinen Fall von Circulus gesehen haben. Auf Güund ron Mosers; Referat läßt Fall 5 jedoch sehr auf Circulus vitiosus schließen; er konnte durch Enteroanastomose geheilt werden. 8 einschlägige Fälle erlebte Calderara6\}; doch ist aus dem Referat nicht genau festzustellen, wie sich die Fälle auf die einzelnen Methoden verteilen. Da er die hintere Gastroenterostomie bevorzugt, dürften auch einige Circulusfälle nach dieser eingetreten sein. Daran reihen sich noch weitere Mitteilungen über Circulus-vitiosus-Fälle nach hinterer Gastroenterostomie: einer von $\mathrm{K}^{\mathrm{a}} \mathrm{p}$ pele $\mathrm{r}^{\mathrm{j}}$ ), ein zweiter

I) HI ercher, Verschluß und Suspension des Pylorus nach Gastrocnterostomie mittels des Lig. teres hepat. Zentralbl. f. Chir. I9I3, II, S. I707.

2) Madsen, A., Über Gastroenterostomietechnik. Hospitalstidende 1912; Nr. I. Ref. Zentralbl. f. Chir. I9ı2, I, S. 865.

3) Scluwarz, D., Zur Technik der Gastrocnterostomie, Orig. Liecknicki viestnik 1902, Nr. 3. Ref. Zentralbl. f. Chir. 1902, S. 503.

4) Dobrotworski, W. I., Zur Frage ron der Gastroenterostomie. VIII u. 422 S. Diss. St. Petersburg 1909. Ref. Zentralbl. f. Chir. 1909, II., S. II 170 .

5) Moser, Referat ron der Arbeit Leroy, De la Gastroenterostomia. Thèse de Paris, G. Steinheil 1902. Zentralbl. f. Chir. 1902, S. 1136.

6) Calderara, A., Risultati immediati e lontani della gastroentero. stomia. Rivista Veneta die science med. XXVIII, Fasc. VIII, I9II, April 30. Ref. Zentralbl. f. Chir. I9I I, II, S. I360.

7) Kappeler, Erfahrungen über Gastroenterostomie. Nachtrag. Deutsche Zeitschr. f. Chir., Bd. 50, S. 319. 
von $\mathrm{F}$ instere $\mathrm{r}^{1}$ ), ein dritter von $\mathrm{K}$ a is e ${ }^{2}$ ) und 7 Fälle aus der Bonner Klinik, die von $\mathrm{Krabbel}$ und $\mathrm{Ge}$ in it $\mathbf{z}^{3}$ ) berichtet wurden. Diese 7 Fälle stellen nur schwere Störungen im Sinne des echten Circulus vitiosus dar und machen 4, I Proz. des bearbeiteten Materials aus; 2 von diesen Fällen kamen zum Exitus, die anderen wurden durch Operation geheilt. Diese Zahl stellt einen verhältnismäßig hohen Prozentsatz dar. Vergleicht man damit die Resultate unserer Statistik, so erlebten wir bei der vorderen Gastroenterostomie bei gutartigen Magenerkrankungen (bei $\mathrm{Krabbel}$ und Geinitz handelt es sich um eine Zusammenstellung nur gutartiger Erkrankungen) Fälle von Zirkulationsstörungen im Gebiete der Gastroenterostomie in 3,26 Proz. Dabei sind die Fälle mit nachgewiesenem technischen Fehler mitgerechnet. Billigerweise werden diese jedoch abgerechnet werden dürfen, so daß wir für unsere Statistik die Häufigkeit des Circulus vitiosus bei der vorderen Gastroenterostomie der gutartigen Erkrankungen auf 2,6I Proz. berechnen. Wollen wir alle Beobachtungen von Circulus auf die Gesamtzahl unserer Fälle berechnen, so kommen wir auf einen Prozentsatz von 3,47 Proz., die Fälle mit nachgewiesenem technischen Fehler eingerechnet; ohne diese auf einen Prozentsatz von 2.43 Proz.

Aus vorstehender Zusammenstellung sehen wir also, daB auch die hintere Gastroenterostomie vor einem Circulus. vitiosus nicht schützt, auch dann nicht, wenn sie mit allerkürzester Jejunumschlinge angelegt ist, und daß auch dann noch Fälle von echter Spornbildung beobachtet sind. Betrachten wir diese Tatsachen aus der Literatur und dazu die guten Erfahrungen, die wir und andere im allgemeinen mit der vorderen Gastroenterostomie gemacht haben, so erscheinen die vielen Einwände, die gerade in dem Punkt "Circulus vitiosus" gegen die vordere Methode der Gastroenterostomit erhoben worden sind, doch wohl nicht gerechtfertigt.

I) Finsterer, H., Chronischer Circulus vitiosus nach Gastroenterostomie nach Einklemmung von Dünndarmschlingen im Mesocolonschlitz. Bruns' Beiträge z. klin. Chir. I9I2, Bd.81, S.341. Festschrift für v. Hacker.

2) $\mathrm{Ka}$ is e 1 , Uber die Erfolge der Gastroenterostomie. Deutsche Zeitschr. f. Chir., Bd. 6 I, H. 3 u. 4.

3) Krabbel und Geinitz, Beiträge zur Chirurgie der gutartigen Magenerkrankungen. Grenzgebiete 1914, Bd. 27, S. go2. 
Damit soll natürlich nicht gesagt sein, daß die vordere Gastroenterostomie die einzige brauchbare Methode ist, vielmehr wird man immer nach dem jeweiligen Befunde handeln müssen und die Fälle aussuchen, bei denen die vordere und bei denen die hintere Methode anzuwenden ist. Wir müssen uns aber gegen die Behauptung wenden, die von so vielen Chirurgen aufgestellt wurde, daß3 die Gastroenterostomia ant. ant. eo ipso schlechtere Resultate gäbe. Daran ändert auch die Tatsache nichts, daß hin und wieder auch jetzt, jedoch ganz in ähnlicher Weise wie bei der Gastroenterostomia retrocol. post., bei der vorderen Methode Fälle von Circulus vitiosus beobachtet werden, wie in den oben angeführten eigenen Beobachtungen und vielen anderen Fällen atss der Literatur, die ich im einzelnen nicht anführen möchte.

Die rordere Gastroenterostomie ist mit am meisten wegen der Gefahr der Spornbildung, die durch paralleles Herunterhängen der Schenkel der Gastroenterostomieschlinge hervorgerufen wird, von vielen verlassen worden. Aber gerade dieses Moment kann in den allermeisten Fällen durch die Kappelersche ${ }^{1}$ ) Aufhängung rermieden werden, auf die in der hiesigen Klinik ganz besonderer Wert gelegt wird und auf die wir nochmals hinweisen wollen. Diese von $\mathrm{Kappeler}$ vorgeschlagene Technik wird scheinbar von vielen nicht so beachtet, wie es für das gute Gelingen der vorderen Gastroenterostomie erforderlich ist. Wenn in ganz sektenen Fällen trotz dieser Technik doch ein Fall von Spornbildung vorkommen sollte, so könnte er höchstens nur in dem Sinne gegen die vordere Methode der Gastroenterostomie ins Feld geführt werden, wie die oben angeführten Fälle von Circulus vitiosus bei hinterer Gastroenterostomie gegen die hintere Methode. Keine Methode der Gastroenterostomie ist eben so sicher, daB sie Komplikationen ganz vermeiden läßt. Insbesondere sind wir in allen Fällen, in denen Adhäsionen oder motorische Insuffizienz des Magens bei der Entstehung des Circulus vitiosus eine Rolle spielen, was die prophylaktische Vermeidung der Komplikation nach der Gastroenterostomie anbetrifft, weder bei der einen noch der anderen Methode besser gestellt. Ja, man ist unter Umständen sogar

1) Kappeler, O., Erfahrungen über Gastroenterostomie. Dentsche Zeitschr. f. Chir. Bd. 79, S. 113. 
besser daran, wenn man bei eingetretener Komplikation an einem Befund bei vorderer Gastroenterostomie als bei hinterer herangehen kann, um eine Enteroanastomose usw. auszuführen. Darüber sind aber schon oben einige kurze Bemerkungen gemacht worden. $\mathrm{DaB}$ die klassische Wölflersche Methode der Gastroenterostomia ant. ant. technisch einfacher ist als die retrocolica, steht außer Frage.

Ich darf zum Schluß noch ein paar kurze Bemerkungen zu den anderen im Anschluß an die Gastroenterostomie auftretenden Passagestörungen machen. Die Gefahr einer Kompression des Colon durch die vor dasselbe gezogene Jejunumschlinge oder die Kompression dieser durch ein stark geblähtes Colon ist nur gering, wenn man die Jejunumschlinge lang genug nimmt, also etwa $30-40 \mathrm{~cm}$ lang. Bei starker Adipositas und sehr fett. reich em Netz oder bei sehr tief stehendem Colon transversum wird die Kompressionsgefahr natürlich größer sein, so daß hier einige der Ausnahmen vorliegen dürften, in denen allgemein die hintere Gastroenterostomie vorzuziehen ist.

Der Gefahr des Durchschlupfes eines Teils der Dünndarmschlingen durch den zuführenden Schenkel der Gastroenterostomieschlinge muß man gewärtig sein, der darauf folgende Ileus wird häufig Ursache zu einer erneuten Laparotomie geben. Eịnen solchen Fall konnten auch wir beobachten. Es handelte sich um einen 57jähr. Zimmermann H. P., bei dem wegen inoperablen Carcinoms und Pylorusstenose eine vordere Gastroenterostomie gemacht wurde. Wenige Tage nach der Operation wurde der Leib aufgetrieben, Patient klagte über starke Leibschmerzen. Winde und Stuhl waren verhalten. Exitus an zunehmender Schwäche. Die Obduktion ergab einen stark geblähten Dünndarm, dessen Schlingen blau verfärbt waren. Bei genauer Inspektion zeigte sich, daß schon im obersten Teil des Dünndarms derselbe total hinter dem zuführenden Schenkel der Gastroenterostomie hindurchgeschlüpft wár und hierdurch eine Strangulation des Dünndarms und in ihrer Folge ein paralytischer Ileus entstanden war. Solche und ähnliche Fälle von Ileus kommen nicht nur bei der vorderen Gastroenterostomie, sondern ebenso nach der hinteren vor, wie ein Fall von Finsterer (1. c.) beweist, bei dem nicht nur die Erscheinungen eines echten Circulus vitiosus bestanden, sondern 
eine Dünndarmschlinge durch den Mesocolonschlitz hindurchgeschlüpft und dort eingeklemmt war. Ein zweiter Fall wird von. Barker1) mitgeteilt, der fast genau dem unserigen gleicht und bei dem es sich um einen Durchschlupf hinter die zuführende Schlinge einer hinteren Gastroenterostomie handelte. Ein dritter ähnlicher Fall wurde mir durch mündliche Mitteilung von Herrn Dr. Eggers (früher Assistent an der Bonner Klinik), bekannt, bei dem es sich auch wieder um einen Durchschlupf von Teilen des Dünndarmes hinter dem zuführenden Schenkel einer hinteren Gastroenterostomie gehandelt hatte.

Es würde zuweit führen und dem Rahmen dieser Arbeit nicht entsprechen, des näheren darauf einzugehen, welche Momente noch weiter gegen und für die eine oder andere Methode der Gastroenterostomie ins Feld geführt, welche Modifikationen der Gastroenterostomie - es sind ihrer sehr zahlreiche - zur Vermeidung der Komplikationen vorgeschlagen worden sind. Aus vorstehendem geht aber das wohl zur Genüge hervor, daß zum mindesten, wie auch von anderer Seite mehrfach anerkannt worden ist, keine der Methoden der Gastroenterostomie die besprochenen Komplikationen ganz vermeiden läßt. Die vordere Wölflersche Methode der Gastroenterostomie war wegen ihrer Komplikationen in Verruf gekommen. Man bedachte dabei aber nicht, daß sie die einzig geübte Methode war und deshalb die Komplikationen nur bei ihr auftreten konnten. Als die hintere Methode der Gastroenterostomie gefunden und immer mehr ausgeführt wurde, da mehrten sich auch die Beobachtungen von ganz gleichartigen Komplikationen im Anschluß an diese Methode; und jetzt, wo sie die am häufigsten geübte Art der Gastroenterostomie ist, liest man viel häufiger über Komplikationen, die in ihrem Anschluß auftreten, als bei der alten Methode der Gastroenterostomie.

So glauben wir behaupten zu können, daß die beiden hauptsächlich angewendeten Methoden der Gastroenterostomie, die antecolica anterior und retrocolica posterior, sich in bezug auf ihre Brauchbarkeit und ihre Komplikationen (Ulcus pepticum,

I) Barker, A. E., A remarkable sequel to a case of Gastroenterostomy, Lancet 1904, Nov. ;. Ref. Zentralbl. f. Chir. I904, Bd. 2, S. I 500. 
Circulus vitiosus, Ileus) zum mindesten die Wage halten, ja, daß die vordere Gastroenterostomie noch den Vorteil der schnelleren und aseptischeren Ausführbarkeit hat, sowie ein leichteres Operieren bei eingetretener Komplikation gestattet als die hintere Gastroenterostomie.

Man hat die hintere Gastroenterostomie als die physiologischere Methode bezeichnet. Das dürfte zutreffen. Andererseits fällt.eine Ausschaltung eines Darmabschnittes von $30-40 \mathrm{~cm}$ gegenüber einer solchen von 5-Io $\mathrm{cm}$ kaum ins Gewicht, auch nicht, wie oben dargetan, in der Frage des Ulcus pepticum. Nimmt man allerdings eine viel längere Schlinge $-50-75 \mathrm{~cm}-$, wie bei $\mathrm{Katr}^{1}$ ) angegeben ist, so fällt die Ausschaltung dieses verhältnismäßig langen Darmabschnittes schon mehr ins Gewicht.

Die Gastroenterostomia antecolica anterior gibt auf Grund unserer Erfahrungen, denen sich ähnliche aus der Literatur anschließen, in bezug auf ihre Funktion gute Resultate, und das um so mehr, je genauer die technischen Momente bei der Ausführung derselben beachtet werden. Betonen möchte ich nochmals, daß es gewisse Ausnahmen gibt, in denen lieber die hintere Gastroenterostomie ausgeführt werden soll; sie sind oben bereits kurz angedeutet worden.

Zusammenfassend darf ich deshalb unseren Standpunkt in der Frage der Technik dahin präzisieren, daß die Erfolge mit der vorderen Gastroenterostomie in der hiesigen Klinik zurückzuführen sind

I. auf die Wahl einer Jejunumschlinge von $30-40 \mathrm{~cm}$;

2. auf ihre Anheftung am tiefsten Punkt der Vorderfläche des Magens in isoperistaltischem Sinne;

3. auf die sorgfältige Aufhängung des zu- und abführenden Schenkels in einer Ausdehnung von 5-6 cm nach dem Vorbilde von $\mathrm{K}$ a p peler.

Das größte Gewicht ist auf diese Aufhängung des ab- und zuführenden Schenkels der Gastroenterostomieschlinge zu legen. Nur dank dieser Modifikation der alten Wölflerschen Methode

I) Katz, Die Gastroenterostomia posterior retrocolica verticalis (v. Hacker-Kausch), Beiträge z. klin. Chir. I9ı1, Bd. 7ı, S. 676. 
kann die Spormbildung und damit auch der echte Circulus vitiosus im Sinne von v. Mikulic z in den allermeisten Fällen vermieden werden. Das angeblich häufigere Auftreten der Spornbildung und des Circulus vitiosus bei der vorderen Gastroenterostomie ist nur dadurch zu erklären, daß die Aufhängung nach $K a p$ peler entweder nicht genügend beachtet wird oder vielen gar nicht bekannt ist und in manchen Hand- und Lehrbüchern der Chirurgie überhaupt nicht erwähnt wird. Deshalb möchte ich an dieser Stelle nochmals auf die Angaben von $\mathrm{K}$ appeler hinweisen.

Anmerkung. Während der Korrektur erscheint jetzt die Arbeit von Bundschuh (Beitr. z. klin. Chir., Bd. Ix9, H. r, S. 62), der aus der Freiburger Klinik über 3 Fälle von Circulus vitiosus nach hinterer Gastroenterostomie berichtet. Ich erwähne sie hier noch nachträglich der Vollständigkeit halber. 